\title{
COMPARISON THEOREM FOR TWO-PARAMETER MEANS
}

\section{ALFRED WITKOWSKI}

Abstract. The comparison theorem for

$$
R(u, v ; r, s ; x, y)=\left(\frac{E\left(r, s ; x^{v}, y^{v}\right)}{E\left(r, s ; x^{u}, y^{y}\right)}\right)^{1 /(v-u)}, u \neq v,
$$

where $E$ is the Stolarsky mean, is proved. This generalises the results of Leach, Sholander and Páles.

Mathematics subject classification (2000): 26D15.

Keywords and phrases: Extended mean values, Gini mean, Stolarsky mean.

\section{REFERENCES}

[1] P. Cinder, Zs. PÁLES, Minkowski-type inequalities for two variable Stoalrsky means, Acta Sci. Math. (Szeged)69 (2003), 27-47.

[2] P. CINDER, Zs. PÁlES, Local monotonicity properties of two-variable Gini means and the comparison theorem revisited, J. Math. Anal. Appl. 301 (2005) 427à-438.

[3] E. B. LEACH, M. C. Sholander, Extended mean values, Amer. Math. Monthly 85 (1978), 84-90.

[4] E. B. LEACH, M. C. SHOlander, Extended mean values II, J. Math. Anal. Appl. 92 (1983), 207-223.

[5] Zs. PÁLES, Inequalities for sums of powers, J. Math. Anal. Appl. 131 (1988), 265-270.

[6] Zs. PÁLES, Inequalities for differences of powers, J. Math. Anal. Appl. 131 (1988), 271-281.

[7] Zs. PÁLES, Comparison of two variables homogeneous means, International Series of Numerical Mathematics, vol. 103 Birkhäuser Verlag, Basel (1992), p. 59-70

[8] K. B. STOLARSKY, Generalizations of the logarithmic mean, Math. Mag. 48 (1975), 87-92.

[9] A. WitKowski, Weighted extended mean values, Colloq. Math. 100(1) (2004), 111-117. 